\title{
Storia senza storie (IV-V secolo d.C.)
}

\author{
ANTONIO BALDini \\ Università di Bologna \\ Italia
}

Resumo. História sem histórias (século $I V-V$ d.C.). O autor expõe alguns resultados sobre a Antigüidade Tardia no âmbito da Quellenforschung, em adesão ao tema da Távola Redonda da qual participa. A exposição desses resultados é baseada nas pesquisas pessoais, comparadas com as de outros estudiosos. Os pontos que o Autor acha que devem ser considerados firmes são os seguintes: existiu, realmente, a assim chamada Kaisergeschichte de Enmann, e esta História Imperial era escrita em latim, por um autor de origem grega e de cultura latina, ou seja, Eusébio de Nantes. Essa dupla característica explica os contatos entre a tradição derivada em latim e aquela em grego. Eusébio, de fato, usava principalmente autores gregos, os mesmos que usava, de forma independente, Eunápio de Sardi, cuja primera edição da História era a base do Livro I da História Nova de Zósimo, que é em grego, porém próxima da tradição latina. Da mesma forma, são aceitos os Anais de Nicômaco Flaviano, em latim: seu conhecimento por parte de Eunápio explica sua reelaboração da própria obra histórica, que confluiu na História Nova de Zósimo, a partir do Livro II.

Palavras-chave. Enmann; Kaisergeschichte; Eusébio de Nantes; Eunápio; Zósimo; Nicômaco Flaviano.

Si tratta qui di ripetere, e riprendere in forma scritta in tempi estremamente compressi, quanto detto in occasione del Convegno F.I.E.C. tenutosi a Ouro Preto nell'agosto 2004. Ora come allora, dichiaro l'intenzione non di esporre il risultato di uno studio su di un aspetto particolare nell'ambito della "Quellenforschung sulla storiografia della Tarda Antichità", tema della mia sessione, ma di approfittare dell'occasione offertami per delineare il quadro da me ricostruito - non certo nella sua interezza - circa la situazione della tarda storiografia.

Come banale nozione preliminare, la produzione storiografica in questione è di ambito profano e di segno religioso pagano, nel senso di una deliberata non assimilazione al cristianesimo dominante, con posizioni nei cui confronti, che variano dalla eloquente reticenza fino alla polemica più aspra. Ancora, il periodo della storia imperiale che arriva ai primi decenni del III secolo d.C. è coperto da almeno due storici grosso modo contemporanei ai fatti riferiti, e quindi attendibili(?) da un punto di vista ricostruttivo. 
Ma per il lungo periodo successivo, lo storico più vicino nel tempo ai fatti di III secolo d.C. riferiti è Aurelio Vittore, che scrive una "storia abbreviata" alla metà del IV secolo d.C.; e Aurelio Vittore sarà seguito da altri sul genere, come Eutropio, Festo, l'anonimo autore dell'Epitome de Caesaribus, o in campo cristiano Gerolamo nel suo Chronicon, ed Orosio, le cui Historiae adversus paganos, pur così ideologicamente orientate, hanno la stessa struttura fattuale dei corrispettivi pagani. E poi, il IV secolo d.C., dalla sua seconda metà, è coperto dal quasi contemporaneo Ammiano Marcellino, che però, se non altro dal punto di vista delle informazioni, non è lonely (come in altra accezione definiva Momigliano), ma gli si deve affiancare la Storia Nuova di Zosimo, che percorre con aumento progressivo della scala narrativa la storia imperiale romana a partire da Augusto fino alla vigilia del sacco di Roma da parte di Alarico, superando quindi gli estremi ammianei all'inizio e alla fine. Ma Zosimo, che assai sovente si mostra impermeabile ad Ammiano Marcellino, è storico dell'età di Anastasio, primi VI secolo d.C., come rilevò tempo addietro Alan Cameron, e come è stato confermato più recentemente': quindi doveva avere qualche fonte (non Ammiano, all'evidenza testuale) a disposizione. Il fatto, ancora, è poi che Zosimo fino alla fine — che, si badi, non è quella voluta, ma imposta da circostanze accidentali ${ }^{2}$ - mostra contatti con tradizione latina non bene identificabile: e quindi si giustifica così il titolo "Storia senza storie", in quanto appare chiaro che le nostre informazioni per il periodo derivano da storici lontani nel tempo, condizionati da altre opere ora perdute, ma comunque da postularsi all'origine delle informazioni stesse.

Allo stato, occuparsi di tarda storiografia comporta un'operazione preliminare di Quellenforschung, senza la quale inficiare il risultato è praticamente certo; e si confrontino le osservazioni di Paschoud e sulla scia di Ratti $^{3}$, nel cui solco mi pongo deliberatamente, e con la piena consapevolezza che nell'intero assieme di tutti questi tardi autori, conservati o perduti, nessuno può essere considerato singolarmente, avulso da rapporti con gli altri, nemmeno tenendo come discriminante il criterio della differenza nell'espressione linguistica, latina o greca ${ }^{4}$.

${ }^{1}$ A. Cameron, The Date of Zosimus' New History, Philologus, 113, 1969, p. 106-110; e cf. F. PAschoud, in Zosime, Histoire Nouvelle, I, livres I-II, texte établi et traduit par F. P., Nouvelle édition, Paris 2000, p. VII-XX.

${ }^{2}$ Paschoud, Zosime, Histoire Nouvelle, I, cit. a n. 1, p. xxv-xxvI.

${ }^{3}$ Paschoud, Quelques problèmes actuels relatifs à l'historiographie de l'antiquité tardive, SO, 73, 1998, p. 74-87; S. RATTI, L'historiographie latine tardive, III ${ }^{e}-I V$ siècle. État des recherches 1987-2002, Pallas, 63, 2003, p. 209-232.

${ }^{4}$ Una condivisibile critica preliminare è stata volta da R.W. BURGESS in una recensione 
Ed ora, l'avvertenza che segue non vuole essere presa come espressione di falsa modestia o come excusatio di pigrizia, ma come adeguamento alla sede di pubblicazione nel rispetto dei curatori: gran parte della mia attività scientifica è stata dedicata a questi temi di tarda storiografia, e ho prodotto un certo numero di lavori brevi e anche saggi di una certa mole; eppure, mentirei se dicessi di avere raggiunto una qualche certezza, tranne che su aspetti minuti. Nel complesso, ogni singolo problema ha una sua consistenza di termini e di dottrina; ma ogni singolo problema appunto è collegato ad un altro alla sua volta fornito di consistenza, in maniera che da qualunque punto si parta, si produce una sorta di effetto domino. Lo stato globale della questione sulla tarda storiografia profana, con storicizzazione di ogni punto specifico nell'ansia di informare (e di fare mostra di essere informati) ${ }^{5}$ comporta un dispendio degno di colossale monografia, non certo di lavoro compreso tra gli Atti di un Convegno di stampo 'universale' sul mondo classico. La via che ho scelto per queste poche pagine, è nell'assumere l'atteggiamento di chi parli ad iniziati, non certo dunque per superbia, ma solo per semplicità, cercando di ridurre al massimo i rinvii bibliografici, e facendovi ricorso solo quando funzionali al discorso. Lo scopo, è proporre la pallida immagine che mi sto figurando circa parte della storiografia profana tardoantica; questa immagine, è indotta da una serie di studi particolari, miei e di altri (la cui conoscenza è data per scontata): del resto, è evidente che nessuno oggi può procedere da solo in un terreno vergine, e terreni vergini in senso stretto non ce ne sono praticamente più. Ogni risultato, anche minimo o settoriale o parziale, è prodotto da confronto tra opinioni personali del singolo con quelle di colleghi o predecessori; con questa consapevolezza, procederò come se tutto fosse frutto di mia indagine personale, ma in realtà poche sono le mie idee originali, e molte quelle di altri: io cerco solo di metterle insieme ed esporle secondo quella che a me pare la costruzione più coerente.

\section{La Kaisergeschichte di Enmann}

Ricordando che qui si è in sede di Quellenforschung, un primo dato che darei per acquisito, è quello dell'esistenza di una perduta Storia Imperiale

telematica (http://ccat.sas.upenn.edu/bmcr/) a Greek and Roman Historiography in Late Antiquity. Fourth to Sixth Century A.D., ed. by G. Marasco, Leiden-Boston 2003: la distinzione operata nel titolo (e la conseguente partizione tra i vari saggi costitutivi) non rende ragione della omogeneità culturale, e soprattutto dell'intreccio delle influenze reciproche tra opere di lingue diverse.

${ }^{5}$ Vorrebbe rispondere a quest'istanza il volume curato da MARASCO, cit. a n. 4 , cui corre l'obbligo di rinviare. 
(Enmanns Kaisergeschichte) già disvelata da Alexander Enmann ${ }^{6}$ nel 1884. È sufficiente riflettere sulla distanza nel tempo tra la fine di Cassio Dione ed Erodiano ed il periodo di composizione del primo storico noto e pervenuto, cioè Aurelio Vittore (metà del IV secolo d.C., ed oltre), il quale a sua volta (e con lui gli "emuli": Eutropio, Festo, Gerolamo anche se sui generis, Epitome de Caesaribus, e parte corposa della Historia Augusta7) copre senza soluzione di continuità la catena dei fatti di III sec. d.C. (e prima, ed oltre), per essere costretti ad ammettere l'esistenza di una qualche fonte scritta. Con questa, si spiegano le somiglianze, ma soprattutto si giustificano le aggiunte o le differenze tra l'uno e l'altro dei cosiddetti "Abbreviatori" per episodi comuni. Ad una tendenza, per altro abbastanza isolata, a sostituire ad una sola Kaisergeschichte più filoni interpretativi ${ }^{8}$, si oppone la difficoltà costituita dalla moltiplicazione delle incognite. Val meglio la risistemazione con la definizione della cronologia di composizione negli anni '50 del IV secolo d.C., la conferma della matrice gallica, ed il suggerimento di Eusebio di Nantes come autore dell'opera'. Eusebio di Nantes è una sorta di ipostasi onomastica, comunque e se non altro, per un autore di radicamento in Gallia (Nantes), ma di estrazione greca (Eusebio), che permette di risolvere un'aporia delle nostre conoscenze in ambito di storiografia: infatti, accanto alle considerazioni di ordine generale sopra esposte, ne sta una più particolare ma non meno importante, per cui, con l'eccezione di qualche bogus name dell'Historia Augusta, il III secolo d.C. è privo di storici latini noti di e per il periodo. Diversamente, ne compare un certo numero di lingua greca, almeno attraverso il testimonium di Evagrio Scolastico di Epifania, di cui non è lecito dubitare ${ }^{10}$ : sicché la Kaisergeschichte eusebiana (di Eusebio di

${ }^{6}$ Eine verlorene Geschichte der römischen Kaiser und das Buch De Viris Illustribus Urbis Romae. Quellenstudien, Philologus, Suppl. Band IV, 1884, p. 355-501.

${ }^{7}$ La Historia Augusta è nella mia stessa tavola rotonda trattata da Anthony Birley, cui rinvio ora e per sempre in queste pagine.

${ }^{8}$ A titolo di esempio, G. ZECCHINI, Qualche ulteriore riflessione su Eusebio di Nantes e l'EKG, HAC Genevense, 2, Bari 1999, p. 331-344; ma cf. anche La storiografia greca dopo Dexippo e l'Historia Augusta, HAC Maceratense, Bari 1995, p. 297-310.

${ }^{9}$ R.W. BuRgess, Principes cum tyrannis: Two Studies on the Kaisergeschichte and Its Tradition, CQ, 43, 1993, p. 491-500; On the Date of the Kaisergeschichte, CPh, 90, 1995, p. 111-128; Jerome and the Kaisergeschichte, Historia, 44, 1995, p. 349-369; Jerome's Chronici Canones, Quellenforschung and Fourth-Century Historiography, HAC Argentoratense, Bari 1998, p. 83-104; e già J.-P. CALlu, D'Évagre à l'Histoire Auguste, HAC Genevense, 1, Bari 1994, p. 71-87, partic. p. 77-80; e poi Histoire Auguste, tome V, lere partie, Vies d'Aurélien, Tacite, texte établi, traduit et commenté par F. PAschoud, Paris 1996, p. XLI-XLII, con PASchoud, Noms camouflés d'historiens du 4e siècle dans l'Histoire Auguste, Historia, 44, 1995, p. 502-504.

${ }^{10} \mathrm{Il}$ testimonium di Evagrio fa parte di un ben più lungo panorama sulla storiografia a lui anteriore redatto dall'Ecclesiastico, in cui i predecessori vengono citati secondo la cronologia dei tempi coperti, e non di quelli di vita invero; comunque, cf. Euagr., $H E, 5,24$, p. 
Nantes) potrebbe acquistare - per quella parte di III secolo d.C. senza riscontri possibili, almeno - una sua plausibilità in quanto scritta in latino (e quindi diventando accessibile agli Abbreviatori), con base su materiale greco, cioè scritto in questa lingua.

Lasciato nella penombra Eusebio di Nantes, ma ricordando l'importanza di una figura che comunque ha fatto da tramite tra la storiografia in lingua greca di e per il III secolo d.C., si accentua l'insistenza sul testimonium di Evagrio, che apre in collegamento ad un altro tema: altra fonte importante per la storia imperiale, ma soprattutto per il III secolo d.C., è infatti la Storia Nuova di Zosimo, storico dell'età di Anastasio. Ora, per questa parte Zosimo mostra una serie di contatti con gli Abbreviatori, che non si possono spiegare con una conoscenza diretta di ciascuno di loro partitamente e per minuzie, diciamo, dato il suo modo di rapportarsi con le sue fonti.

\section{Zosimo e le sue fonti}

Sul tema, sono chiamato direttamente in causa ${ }^{11}$, perché ho creduto di aderire all'ipotesi oggi praticamente maggioritaria e quasi mai più discussa ${ }^{12}$ di una assoluta dipendenza di Zosimo da Eunapio di Sardi (fino a 5,25) ed Olimpiodoro di Tebe (da 5,26 alla fine). È infatti tassativa la testimonianza del patriarca Fozio, secondo cui «... qualcuno potrebbe dire che egli (scil.: Zosimo), non ha scritto una Storia, ma trascritto quella di Eunapio, (Storia) che si differenzia soltanto per la concisione...»: se dubbi hanno potuto in-

218,31-219,10 Bidez-Parmentier: «(dopo Cassio Dione ed Erodiano)... avendone poi scritto Nicostrato, il sofista di Trapezunte, risultano esposte anche le cose a partire da Filippo, quello dopo Gordiano, fino ad Odenato di Palmira ed alla vergognosa andata di Valeriano presso i Persiani. Su queste cose ha speso moltissima fatica anche Dexippo, prendendo inizio dalle cose mitiche e terminando al regno di Claudio, quello dopo Gallieno; a queste sono incorporate le cose su ciò che fecero i Carpi ed altri popoli barbari combattendo in Ellade ed in Tracia e Ionia. Ed Eusebio conducendo la narrazione da Ottaviano e Traiano e Marco si spinge fino alla morte di Caro. Intorno a questi tempi sono state scritte alcune cose anche da Arriano e Asinio Quadrato...».

${ }^{11}$ Ricerche sulla Storia di Eunapio di Sardi. Problemi di storiografia tardopagana, Bologna 1984; Le due edizioni della Storia di Eunapio e le fonti della Storia Nuova di Zosimo, AFLM, 19, 1986, p. 47-109. Non so quanto consigliare il saggio dedicato alla storiografia pagana da J.H.W. LieBESCHUETZ in Greek and Roman Historiography, cit. a n. 4, p. 177-218, per via di alcune mende bibliografiche; ma cf. almeno PASchoud, Zosime, Histoire Nouvelle, I, cit. a n. 1, p. XLIII-XLIV ; e già Zosime, Histoire Nouvelle, III, $2^{\mathrm{e}}$ partie, livre VI et index, texte établi et traduit par F. P., Paris 1989, p. 91-94.

${ }^{12}$ Un'eccezione, da segnalare per la possibilità di diffusione data la sede di pubblicazione, è stata G. Fowden, The Last Days of Constantine: Oppositional Versions and Their Influence, JRS, 84, 1994, p. 146-170; e contra, correttamente, PASchoud, Zosime et Constantin. Nouvelles controverses, MH, 54, 1997, p. 9-28. 
sorgere, è perché nelle edizioni dei frammenti eunapiani (Müller o Blockley, ad esempio), tra i frammenti provenienti dagli Excerpta costantiniani (de legationibus, e più de sententiis), che sono il principale tramite di trasmissione, con una sorta di deviante horror vacui vengono inseriti brani dalle Vitae Sophistarum dello stesso Eunapio e lemmi del lessico Suda. Assimilare questo secondo tipo di testimonianze al primo, significa non tenere conto della forza della legge dei generi, che obbligava ad esprimere in moduli narrativi diversi uno stesso episodio a seconda delle opere ${ }^{13}$, e significa al contempo facilitarsi la caduta nell'equivoco. Diversamente, un confronto testuale tra la Storia Nuova ed i frammenti traditi dagli Excerpta depone per una completa dipendenza di Zosimo da Eunapio, con unica differenza nello stile, retorico ed ampolloso quello del secondo, asciutto fin quasi all'incomprensione dell'originale quello del primo, esattamente corrispondendo al giudizio di Fozio $^{14}$. Quanto ad Olimpiodoro di Tebe, la sua presenza nella Storia Nuova è ammessa inconsapevolmente da Zosimo stesso, che a 5,27 lo cita per contraddirlo, secondo costume di questa tarda storiografia di criticare chi si sta seguendo come fonte ${ }^{15}$. Quando è del resto possibile, un confronto tra la prosa di Zosimo ed i cosiddetti frammenti di Olimpiodoro conferma lo stretto rapporto tra i due, con l'avvertenza nel confronto che la trasmissione di Olimpiodoro non è per frammenti del tipo di quelli eunapiani (tramite excerpta), ma deriva dalla recensione che ne ha fatto il patriarca Fozio al codice 80 della sua Bibliotheca, sottoponendo il testo originale all'arbitrio del suo modo di lettura e riferimento ${ }^{16}$. Punto fermo, è dunque che Zosimo vale per le fonti che usa, e di cui è portatore, e che si lasciano individuare esclusivamente nelle opere storiche (perdute) di Eunapio di Sardi e di Olimpiodoro di Tebe.

Questa nozione deve essere trattenuta, in quanto è pilastro dell'intera costruzione, e certo dà adito a problemi, ma contemporaneamente permette di proporne delle ipotesi di soluzione. Dunque, quanto è noto da Zosimo fino ai primi anni del V secolo d.C. deriva dalla Storia di Eunapio di Sardi.

${ }^{13}$ Già Arnaldo Momigliano, in L'età del trapasso fra storiografia antica e storiografia medievale (320-550 d.C.), "Quinto contributo alla storia degli studi classici e del mondo antico", Roma 1975, p. 49-71, aveva proposto questa istanza di metodo, e cf. più in specifico la ripresa di PAschoud, La préface de l'ouvrage historique d'Eunape, Historia, 38, 1989, p. 198-223, partic. p. 210-213, e J.A. ОсноA, La transmisión de la Historia de Eunapio, Erytheia — Estudios y Textos, 1, Madrid 1990, p. 33-37, 40-47.

${ }^{14}$ A. BALDini, Ricerche sulla Storia di Eunapio di Sardi, cit. a n. 11, p. 41-74; e cf. PAschoud, Zosime, Histoire Nouvelle, $\mathrm{III}^{2}$, cit. a n. 11, p. 82-94.

${ }^{15}$ Paschoud, in Zosime, Histoire Nouvelle, I, cit. a n. 1, p. LXV-LXIX, ed anche fino a p. LXXI.

${ }^{16}$ BaLdini, Ricerche di tarda storiografia (da Olimpiodoro di Tebe), Bologna 2004, p. $62-82$. 
E però, il patriarca Fozio, recensendo Eunapio, afferma che la sua Storia cominciava dal 270 d.C., cioè dalla fine dell'opera di Dexippo: «Fu letta la Storia in forma di Cronaca di Eunapio, quella dopo Dexippo, nella nuova

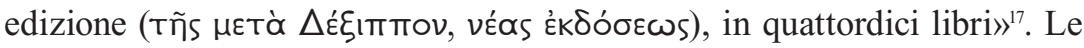
stesse caratteristiche, essere dopo Dexippo e una nuova edizione, appaiono nell'intestazione degli Excerpta costantiniani da Eunapio ${ }^{18}$, ed Eunapio, in un frammento tratto dalla nuova edizione, conferma di avere preso inizio da Dexippo $^{19}$. Il fatto è che Zosimo inizia da prima di Dexippo, cioè da Augusto, e dopo una serie di capitoli in cui la narrazione è estremamente stringata, la scala del racconto si amplia fino a quella di una vera Storia; e soprattutto lo scorrere del racconto non presenta né fratture né soluzioni di continuità. Sorge quindi la questione su a chi abbia attinto Zosimo (così distante nel tempo da quei fatti) per quella parte "prima di Dexippo", questione che a sua volta, come prima anticipato, si collega all'altra su come spiegare i contatti con gli Abbreviatori dovendosene escludere una conoscenza diretta da parte appunto di Zosimo.

Una proposta di soluzione a questa aporia ${ }^{20}$ può essere suggerita da come il patriarca Fozio prosegue nella sua recensione ad Eunapio: «... Egli poi ha composto due opere contenenti la stessa storia, una prima ed una seconda, e nella prima sparge molta diffamazione contro la pura fede di noi cristiani, ed esalta la superstizione ellenica, rivolgendo molte calunnie ai pii imperatori; nella seconda, quella che intitola anche nuova edizione (véav

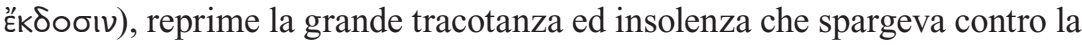
pietà, ed avendo poi connesso il restante assieme della materia storica, lo intitola, come dicemmo, nuova edizione, che ancora mostra molto del livore di quell'altra. Abbiamo trovato queste due edizioni in antichi esemplari: in uno, ciascuna delle due era a parte; nell'altro, erano combinate. Da queste stesse edizioni, alla lettura, abbiamo constatato la differenza tra loro.... $\rangle^{21}$. A prescindere qui da alcuni problemi collaterali posti dal testo di Fozio, resta il

${ }^{17}$ Phot., Bibl., 77, p. 159,34-35 Henry, I.

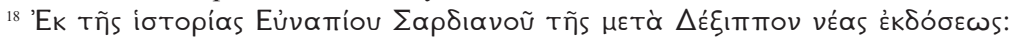
Excerpta de sententiis, 1, p. 71,1-2 Boissevain (è lecito dubitare se il titolo fosse quello voluto da Eunapio, o se non derivasse da caratteristiche dell'opera rilevate dall'escertore).

${ }^{19}$ Eun., fr. 8 Müller, $F H G$, IV, p. 15-16; 15, p. 20,1-3 Blockley; Excerpta de sententiis, 5, p. 76,14-77,7, partic. p. 76,14-16 Boissevain: «Gli avvenimenti dunque che dalla fine della composizione di Dexippo si distendono fino ai tempi di Giuliano sono stati esposti a sufficienza nella parte precedente quanto meglio era possibile per chi correva attraverso le cose necessarie...».

${ }^{20}$ BALdini, Ricerche sulla Storia di Eunapio di Sardi, cit. a n. 11, p. 179-230; e cf. PAschoud, Zosime, Histoire Nouvelle, I, cit. a n. 1, p. XLIII-XLVI.

${ }^{21}$ Phot., Bibl., 77, p. 159,37-160,3, Henry, I. 
nocciolo della sua affermazione: esistere ancora ai tempi suoi (IX secolo d.C.) esemplari di due edizioni tra loro diverse della Storia di Eunapio di Sardi; e l'incipit della sua recensione ad Eunapio indica che ha appuntato l'attenzione sulla seconda, che cominciava da Dexippo, e che la seconda edizione cominciasse da Dexippo è dichiarato dall'intestazione degli Excerpta de sententiis, un frammento eunapiano dai quali conferma di avere preso inizio da Dexippo. A ben guardare, però, non c'è prova che anche la prima edizione cominciasse da Dexippo; e di conserva, Eunapio stesso conferma l'esistenza di una (prima) edizione della sua Storia composta già quando scriveva le Vitae Sophistarum. In più passi ${ }^{22}$, infatti rimanda il lettore ad una propria opera storica, non univocamente definita, per avere maggiori e diverse notizie su un argomento o personaggio trattato nelle Vitae, ed in particolare è interessante VII,5,10-6,13, p. 55 Giangrande, che attesta che la (prima edizione della) Storia comprendeva almeno la morte di Valente ad Adrianopoli ${ }^{23}$. La prima edizione era dunque una realtà, ed esemplari di essa potevano circolare ai tempi di Zosimo, se erano ancora disponibili ben più tardi, ai tempi di Fozio. Scartata già da tempo l'ipotesi che Zosimo potesse avere attinto direttamente a Dexippo per il periodo a partire da Augusto, la prima edizione della Storia di Eunapio come fonte per l'intero libro primo della Storia Nuova diviene un'ipotesi di lavoro seducente, in quanto supportata anche da una serie di considerazioni: e silentio, non si scorge quale storico di lingua greca possa essere stato utilizzato da Zosimo; l'intero primo libro aumenta gradatamente la scala del racconto, ma non mostra alcuna frattura al suo interno, come invece sarà perspicuo nel passaggio poi dalla parte eunapiana $\mathrm{a}$ quella olimpiodorea; nel primo libro della Storia Nuova compaiono una serie di espressioni, anche rare, che appaiono improntate a Giuliano (e quindi al giulianeo Eunapio), e che talora sono in comune tra Giuliano, Eunapio (Vitae Sophistarum) e Zosimo appunto; in una parte sicuramente eunapiana (1,57 e 58) si ha la spiegazione di formulazioni espresse nella parte proemiale, conferendo ad essa una logica altrimenti monca. Del resto, i punti di inizio tradizionali nella storiografia antica (e soprattutto in quella tarda) erano dalla fine di un predecessore illustre, oppure dall'inizio del Principato. La

${ }^{22}$ Paschoud, Eunapiana, BHAC 1982/1983, Bonn 1985, p. 239-303, partic. p. 253-256; OсHOA, La transmisión, cit. a n. 13, p. 29-55.

${ }^{23}$ Qui, il filosofo Massimo predice che «"Dopo la comune e multiforme rovina di tutti (scil.: i filosofi), nella quale saremo oggetto della strage, l'imperatore morirà in modo per così dire sorprendente, neppure stimato degno di cerimonia funebre né di illustre sepolcro".

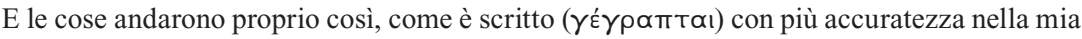
Storia generale... L'imperatore infatti in una grande battaglia contro gli Sciti scomparve in modo strano, così che neppure un osso ne fu trovato nello sgombero dei cadaveri (partic. p. 55, 22-24 Giangrande)». 
seconda edizione, che era un rifacimento ma non uno stravolgimento della prima, iniziava dall'illustre predecessore Dexippo, e nulla vieta di pensare che la prima avesse preso inizio dalla fondazione del Principato, che è di fatto il punto di inizio della narrazione della Storia Nuova. Accettare questa ipotesi, significa rendere ragione delle prossimità, talora, tra Storia Nuova ed Abbreviatori: ricordando la matrice greca dell'autore della Kaisergeschichte di Enmann, che gli aveva permesso di utilizzare gli storici greci noti per il III secolo d.C., questi stessi storici potevano essere stati utilizzati direttamente anche da Eunapio; e tra questi storici poteva essere anche Dexippo, il cui influsso comunque appare evidente nel primo libro della Storia Nuova, pur dovendosi escludere un suo utilizzo diretto e continuato.

\section{Gli Annales di Nicomaco Flaviano senior}

Relativamente a questo problema, ben poco di mio, se non adesione a quanto da più parti si viene elaborando. Questi Annales hanno un'attestazione esplicita in $I L S, 2948$, del 431 d.C., che contiene l'epistola al senato di Roma di Teodosio II e Valentiniano III con cui si riabilitava la memoria di Virio Nicomaco Flaviano senior, compromessosi fino al suicidio nell'usurpazione di Eugenio ${ }^{24}$; ed a questo testo si collega ILS, 2947, in cui il personaggio è qualificato come historicus disertissimus. Anche se le testimonianze letterarie non hanno alcun cenno esplicito, è chiaro che questi Annales non possono essere messi in discussione. Ed infatti, ebbero una loro intuizione (come elaborazione greca di un originale latino) alla fine del $1800^{25}$, una proposta alla ribalta nel classico di Hartke sui Römische Kinderkaiser, ed hanno una loro valutazione in studi recenti, che si possono compendiare nei nomi di Bleckmann, Paschoud, Zecchini, Festy, Ratti ${ }^{26}$. Attraverso la media-

${ }^{24} \mathrm{Al}$ documento epigrafico, ed al tema connesso della riabilitazione di Nicomaco Flaviano senior è dedicato CH. W. Hedrick JR., History and Silence. Purge and Rehabilitation of Memory in Late Antiquity, Austin 2000, da cui per altro poco si cava circa i problemi storiografici posti dall'attestazione degli Annales.

${ }^{25}$ E. PAtZIG, Über einige Quellen des Zonaras, ByzZ, 5, 1896, p. 24-53; ByzZ, 6, 1897, p. 322-356.

${ }^{26}$ B. Bleckmann, Die Chronik des Johannes Zonaras und eine pagane Quelle zur Geschichte Konstantins, Historia, 40, 1991, p. 343-356; Pagane Visionen Konstantins in der Chronik des Johannes Zonaras, in "Costantino il Grande dall'Antichità all'Umanesimo", Colloquio sul Cristianesimo nel mondo antico, Macerata, 18-20 dicembre 1990, I, a cura di G. Bonamente e F. Fusco, Macerata 1992, p. 151-170; Die Reichskrise des III. Jahrhunderts in der spätantiken und byzantinischen Geschichtsschreibung. Untersuchungen zu den nachdionischen Quellen der Chronik des Johannes Zonaras, München 1992; Die Schlacht von Mursa und die zeitgenössische Deutung eines spätantiken Bürgerkrieges, in "Gedeutete Realität. Krisen, Wirklichkeiten, Interpretationen (3.-6. Jh. n. Chr.)”, hrsg. von H. BRANDT, 
zione di Pietro Patrizio - Anonymus post Dionem, percorrono la tradizione bizantina fino a Zonaras, già presenti in distillato in Leo Grammaticus; con questi Annales dialoga ogni tanto Ammiano Marcellino ${ }^{27}$, e soprattutto questi Annales sono presenti nell'ultima parte dell'Historia Augusta, almeno a partire dalla Vita di Aureliano ${ }^{28}$, e l'Historia Augusta è opera che può ben emanare dalle cerchie dei Symmachi-Nicomachi; ma soprattutto, è merito di Michel Festy, e già prima di Jörg Schlumberger, avere mostrato che questi Annales compaiono con una certa frequenza accanto alla tradizione della Kaisergeschichte di Enmann nell'Epitome de Caesaribus, operetta talora sconcertante ma comunque preziosa ${ }^{29}$. Ora, nell'Epitome, a proposito della conversione di Costantino (ma questo è solo un esempio di comodo), compaiono dati estranei alla tradizione enmanniana e vicini invece a quelli di Zonaras - chiaramente, il tramite sono gli Annales di Nicomaco Flaviano senior -, ma soprattutto qui, a quelli del capitolo relativo nel libro secondo della Storia Nuova di Zosimo ${ }^{30}$ : il figlio Crispo fatto mettere a morte per istigazione di Fausta, la morte nel bagno surriscaldato di Fausta per istigazione della madre dell'imperatore Elena... Questo spunto, chiaramente flavianeo, porta ad una proposta risolutiva di una perplessità relativa allo statuto di Zosimo in relazione alle sue fonti, in particolare Eunapio. A partire dal secondo libro, compaiono in Zosimo numerose tracce di una fonte occidentale, anche se non in maniera continuativa, come da più parti sottolineato, ed una di queste è l'ambientazione romana dell'episodio della conversione di Costantino, preceduto dall'abbandono dei ludi saeculares, e seguito ad una certa distanza dal gioco di parole sulla profezia circa la morte di Graziano (pace Cameron, in questa stessa sede). Ed ancora, e più a monte, rimane l'interrogativo su cosa abbia spinto Eunapio a rifare la propria opera storica,

Stuttgart 1999, p. 47-101; PASchoud, Nicomaque Flavien et la connexion byzantine (Pierre le Patrice et Zonaras): à propos du livre récent de Bruno Bleckmann, AnTard, 2, 1994, p. 71-82; G. Zecchini, Da Nicomaco Flaviano a Memmio Simmaco. La fine della storiografia classica in Occidente, in "Ricerche di storiografia latina tardoantica", Roma 1993, p. 51-63; M. Festy, Le début et la fin des Annales de Nicomaque Flavien, Historia, 46, 1997, p. 465478; Ratтi, Jérôme et Nicomaque Flavien: sur les sources de la Chronique pour les années 357-364, Historia, 46, 1997, p. 479-508.

${ }^{27}$ Ad es., Paschoud, Valentinien travesti, ou: De la malignité d'Ammien, in "Cognitio Gestorum. The Historiographic Art of Ammianus Marcellinus", ed. by J. DEN BoEFT, D. DEN Hengst, H.C. Teitler, Amsterdam 1992, p. 67-84.

${ }^{28}$ Ancora a titolo di esempio, PASCHOUd, À propos des sources du récit des campagnes orientales d'Aurélien dans l'Histoire Auguste, HAC Maceratense, Bari 1995, p. 281-295.

${ }^{29}$ J. Schlumberger, Die Epitome de Caesaribus. Untersuchungen zur heidnischen Geschichtsschreibung des 4. Jahrhunderts n. Chr., München 1974; Festy, Pseudo-Aurélius Victor, Abrégé des Césars, texte établi, traduit et commenté par M. F., Paris 1999, p. VII-CV.

${ }^{30}$ Epit., 41,11-12 Festy; Zonaras, 13,2,38-41 Büttner-Wobst; Zos., 2,29,2-3 Paschoud. 
senza cancellare la prima edizione di essa, venendo a costituire un caso pressoché unico nel panorama della storiografia, soprattutto tarda. La risposta potrebbe essere nel confronto con gli Annales di Nicomaco Flaviano senior, ad orientamento ideologico comune, e con una valenza di prestigio dovuta in parte alla fine dell'autore. Così si spiega perché in tutto il libro primo della Storia Nuova non vi siano tracce di storici latini direttamente ${ }^{31}$, mentre queste tracce diventano perspicue nel prosieguo: Zosimo ha usato all'inizio la prima edizione, forse perché cominciava da prima, ed è poi passato alla seconda, forse perché finiva dopo.

\section{Una continuazione della tradizione flavianea?}

Suicidatosi nel 394 d.C., Nicomaco Flaviano senior non può essere più l'autore presente nel cruciale capitolo 59 del libro quarto della Storia Nuova, là dove Zosimo propone un adventus di Teodosio a Roma dopo il Frigido, una sua esortazione ad abbandonare il paganesimo per abbracciare il cristianesimo, una ripulsa del senato che presuppone all'atto di redazione dell'originale la conoscenza del sacco di Roma di Alarico, ed infine una proibizione imperiale dei culti pubblici, con cessazione delle pratiche ancestrali. E la stessa notizia di un adventus teodosiano dopo il Frigido e connessa cessazione delle pratiche ancestrali compare ancora in Zosimo, ma a 5,38, in una parte già di sicura derivazione da Olimpiodoro, storico attento quant'altri mai all'Occidente. Nelle sue Cinq études sur Zosime, del 1975, François Paschoud si era interrogato, ed aveva risposto con un Ignotus autore di una "Storia contro i cristiani" alla luce del sacco del 410. Nel prosieguo, si sono sfumati i termini, ma la sostanza rimane. Ora, anche io mi sono affaticato sul problema, e comunico la mia vicinanza a Paschoud; e tuttavia, quando scrivo queste pagine la mia fatica ${ }^{32}$ non ha ancora avuto riscontri né in positivo né in negativo: non dispongo quindi di materiale su cui riflettere per confermare o smentire le mie sensazioni. Credo dunque sia bene sospendere queste considerazioni, che allo stato rischiano di essere pure speculazioni.

${ }^{31}$ In questo senso, ed a proposito proprio di Nicomaco Flaviano senior, B. GirotTi, $D a$ Evagrio a Zosimo: nota sulle fonti del primo libro della Storia Nuova, RSA, 32, 2002, p. 151-166. Secondo gli spiriti di questo mio lavoro, è deliberata l'omissione della discussione con chi diversamente ritiene Nicomaco Flaviano senior presente fin dall'inizio nella Storia Nuova di Zosimo: ad esempio, Paschoud ispira a questa idea buona parte dell'Introduzione e poi tutto il commento ai libri I-II di Zosimo nell'edizione cit. a n. 1; e già prima, cf. almeno M. MAzZA, La cosiddetta «digressione antimonarchica» in Zosimo, I,5,2-4. Qualche breve nota ed un'ipotesi, in "Synodia Studia humanitatis Antonio Garzya septuagenario ab amicis atque discipulis dicata", a cura di U. Criscuolo e R. MaIsano, Napoli 1997, p. 669-686.

${ }^{32}$ Ricerche di tarda storiografia, cit. a n. 16. 
Ciò che mi premeva, era esporre alcuni punti in apparenza fermi, almeno per me: la Kaisergeschichte di Enmann, la prima edizione della Storia di Eunapio come fonte del primo libro di Zosimo, il comune ma indipendente attingere da parte di Eunapio ed Eusebio di Nantes agli stessi autori greci, l'incidenza degli Annales di Nicomaco Flaviano senior, sia sulla restante storiografia sia soprattutto sullo stesso Eunapio, al punto da indurlo a rifare la propria opera storica; infine il suggerimento che gli spiriti di Nicomaco Flaviano senior, anche dal punto di vista storiografico, non si erano spenti con il suo suicidio.

Deliberatamente, non ho discusso alcuna ipotesi contraria alle mie, al solo scopo di non appesantire inutilmente queste pagine; moltissimi lavori meritevoli di citazione li ho trascurati, ma solo tipograficamente: chi abbia un po' di pratica potrà tranquillamente riconoscerli, e se crede rinfacciarmene l'omissione. Non era questo uno status quaestionis, ma una personale rimessa a punto su temi che hanno accompagnato tanti anni di ricerche.

Abstract. History without histories (iv-v century C.E.). The Author here explains some results he considers acquired within the limits of Quellenforschung of Late Antiquity, to support the theme of the Round Table in wich he is a member. The exposition of theese results is founded on personale researches compared with others studies. Foundamental principles wich the Author wants to consider are the follows: the Enmann's Kaisergeschichte was real existed; that imperial history was written in Latin by a greek author who had a latin education: Eusebius of Nantes. That double peculiarity explains contacts between the tradition delivered in Latin and in Greek. As a matter of fact, Eusebius of Nantes mainly utilized greek authors as sources, the same that indipendently utilized Eunapius of Sardis, whom First Edition of his History was the base of first book of Zosimus' New History, written in greek, but close to Latin tradition. As well, the Author believes in the Annals of Nicomachus Flavianus, written in Latin. The knowledge of them by Eunapius explains his rewriting of his historical work, came together in Zosimus' New History, from the second book.

KeYwords. Enmann; Kaisergeschichte; Eusebio di Nantes; Eunapio; Zosimo; Nicomaco Flaviano. 